\title{
Vertebral Artery Dissection Associated with Generalized Convulsive Seizures: A Case Report
}

\author{
Faisal Mohammad Amin ${ }^{a} \quad$ Vibeke Andrée Larsen $^{b} \quad$ Peer Tfelt-Hansen $^{a}$ \\ ${ }^{a}$ Department of Neurology, and ${ }^{\mathrm{b}}$ Diagnostic Department, Glostrup Hospital, Faculty of \\ Health and Medical Sciences, University of Copenhagen, Copenhagen, Denmark
}

\section{Key Words}

Seizure $\cdot$ Vertebral artery $\cdot$ Dissection $\cdot$ Stroke

\begin{abstract}
A 46-year-old male with juvenile myoclonic epilepsy was admitted to the neurological department for convulsive seizures just after lamotrigine was discontinued. On admission he was awake but had a right-sided hemiparesis with Babinski sign and ataxic finger-nose test on the left side. An MR scan showed a left-sided pontine infarction, an infarct in the left cerebellar hemisphere and a right vertebral artery dissection (VAD). The patient was treated with heparin and an oral anticoagulant for 6 months. Recovery of neurologic function was excellent. In patients with symptoms of disturbances of posterior circulation after epileptic seizures, VAD should be considered.

(c) 2013 S. Karger AG, Basel
\end{abstract}

\section{Introduction}

The refinement of noninvasive neurovascular imaging has established that carotid arterial dissection and vertebral arterial dissection (VAD) are actually the most common causes of stroke in young patients $(<50$ years), accounting for $20-30 \%$ of all ischemic strokes in this age group [1]. The annual incidence of spontaneous VAD is 1-1.5 per 100,000 [2]. Usually, a history of minor blunt trauma or activity associated with rotation or hyperextension of the neck, such as aerobics, yoga, painting of the ceiling and chiropractic manipulations can cause VAD, and it is generally labeled as spontaneous dissection. Traumatic dissection occurs in approximately $1 \%$ of all patients with blunt injury mechanisms and is frequently unrecognized [2]. 
Distraction/extension, distraction/flexion and lateral flexion injuries as well as fractures extending into the transverse foramen or facet joint dislocations are closely associated with vertebral artery injuries [3]. The first documented case of VAD in association with a tonicclonic seizure was published in 1991 [4]. One further case associated with status epilepticus was published in Spanish [5].

VAD secondary to generalized convulsive seizures is not mentioned in the major reviews of cervical arterial dissection [1, 2, 6-8]. This indicates that the condition is rare and may often go unrecognized due to an asymptomatic course of VAD in many patients with unilateral damage [3]. The muscular forces involved in the tonic-clonic phase of convulsive seizures are demonstrated by the occurrence of bone fractures in the spinal column and proximal skeleton [9]. Various fractures have been noted as resulting from convulsive seizures, including fractures of the vertebrae, pelvis, femoral neck and shoulder [9-14]. Most vertebral fractures are compression fractures, but burst fractures with neurological complications can occur [15]. Quadriplegia can result from fracture with dislocation of the cervical column [16]. The fact that muscular forces during a convulsive seizure can cause diverse bone fractures makes it more likely that it can also cause VAD.

Here, we present a case of VAD in connection with a generalized convulsive seizure. The VAD caused embolic complications: pontine and cerebellar infarctions.

\section{Case Report}

An otherwise healthy 46-year-old male engineer, with juvenile myoclonic epilepsy, was admitted to our department following convulsive seizures 1 month after discontinuation of lamotrigine. He was diagnosed with juvenile myoclonic epilepsy at the age of 39 after having 3 generalized convulsive seizures over 3 months. An EEG and brain CT were both normal. He was started on valproic acid $900 \mathrm{mg}$ and was shifted to lamotrigine $200 \mathrm{mg}$ daily 3 years later. After a 5-year period without seizures, lamotrigine was slowly tapered off and the patient completely stopped the intake 1 month before the present admission. He was admitted to hospital with a right-sided hemiparesis with Babinski sign, a right-sided central facial palsy and an ataxic finger-nose test on the left side, which developed following a generalized convulsive seizure 1 day prior to admission. He had no neurological or other complaints up to the seizure. Brain CT was normal, but an MRI scan showed an infarct in the left cerebellar hemisphere (fig. 1a) and a left-sided pontine infarction (fig. 1b). Subsequent MR angiography showed a right VAD (fig. 2a).

In addition, the blood samples for kidney and liver function, haemoglobin and coagulation factors were all in the normal range of reference. However, there was a high leucocyte count with an overabundance of neutrophils. Moreover, the patient's blood pressure at the time of admission was normal $(127 / 87 \mathrm{~mm} \mathrm{Hg})$, and he was afebrile. The patient was started on heparin and the oral anticoagulant warfarin, which he continued for 6 months (from January 10 until June 21). The treatment with lamotrigine was also resumed. Recovery of neurologic function was excellent, and he was discharged after 14 days. A control MR angiography 6 months later showed a normal vertebral artery (fig. 2b). The patient still continued treatment with aspirin and dipyridamole. 


\section{Discussion}

The anatomy of the vertebral arteries (ascendance parallel to the spine through the transverse foramina of the C1-C6) makes them vulnerable to stretching and compression of the spine, especially at the $\mathrm{C} 1 / \mathrm{C} 2$ levels, where arteries are relatively fixed and where a big part of head rotation occurs.

The vertebral arteries wind around the C1 vertebra, so they would be expected to be more susceptible to dissection most likely caused by hyperextension than the carotid artery, which lies more loosely and plainly in the soft tissue of the neck [1]. A generalized tonicclonic seizure involves tremendous muscle contraction and rigidity and clonic movements of the whole body. In particular, the neck region is vulnerable, where hyperextension, rotation, flexion and distraction movements can occur, which are known to cause VAD $[17,18]$. Trauma due to falls can also cause neck trauma, which in turn can cause damage to the vertebral arteries.

Our patient, who had a history of juvenile myoclonic epilepsy and had been seizure free for 5 years, had just recently stopped taking medication. He was admitted to the hospital with generalized clonic seizures and afterwards had a right-sided hemiparesis. The differential diagnosis was Todd's paresis, but the patient had never experienced postictal paresis before. Despite a normal CT scan on admission, we suspected that he had had a minor cortical stroke in the left cerebral hemisphere, which had resulted in a generalized seizure. An MRI was performed 6 days after admission and showed pontine and cerebellar infarctions and right VAD. The differential diagnosis of pontine infarct with quadriplegia is a cervical fracture resulting from hyperextension associated with a fall during an epileptic seizure [16].

Among epileptic patients who have had at least 1 seizure during the previous year, 24\% incurred at least 1 head injury and 6\% suffered some other fractures [19]. Apart from these common injuries associated with epilepsy, rarer complications may occur. Compression fractures and more rarely burst fractures of the vertebrae [15] and fractures of proximal bones of the skeleton are associated with convulsive seizures [16].

In conclusion, epileptic patients who normally do not have Todd's paresis after a generalized convulsive seizure or patients suspected of Todd's paresis but with a duration of $>24 \mathrm{~h}$ should be investigated with neuroimaging for stroke. Stroke-induced generalized convulsive seizure can be the first symptom of a stroke [20]. This is often seen (39\%) in connection with cerebral venous and dural sinus thrombosis or cortical venous infarction [21]. In patients with symptoms of disturbances of posterior circulation after generalized tonic-clonic seizures, VAD has to be taken into consideration. Neurological symptoms can initially be occult and may occur some hours or days later. It is important to diagnose VAD and to treat it in time to avoid further progression and thromboembolic complications. Thromboembolisms are most often a cause of progressive ischemic cerebral symptoms in younger patients and can, in some cases, cause a fatal outcome (in cases of unrecognized thrombosis of the basilar artery).

\section{References}

1 Menon RK, Norris JW: Cervical arterial dissection. Current concepts. Ann NY Acad Sci 2008;1142:200-217.

-2 Redekop GJ: Extracranial carotid and vertebral artery dissection: a review. Can J Neurol Sci 2008;35:146152.

-3 Inamasu I, Guiot BH: Vertebral artery injury after blunt cervical trauma: an update. Surg Neurol 2006;65:238-246. 
Amin et al.: Vertebral Artery Dissection Associated with Generalized Convulsive Seizures: A Case Report

4 Young CA, Chadwick DW, Humphrey PR: Extracranial vertebral artery dissection following tonic clonic seizure. J Neurol Neurosurg Psychiatry 1991;54:365-366.

5 Nunes-de Oliveira S, Moniz JC, Bandeira-Costa JC: Status epilepticus. Dissection of the vertebral artery and ischaemic vascular accident in the pons. Rev Neurol 2007;44:635-636.

-6 Pavel RR, Adam R, Maldjan C, Lincoln CM, Yuen A, Aneja A: Cervical carotid artery dissection: current review of diagnosis and treatment. Cardiol Rev 2012;20:145-152.

7 Goyal MS, Derdeyn CP: The diagnosis and management of supraaortic arterial dissections. Curr Opin Neurol 2009;22:80-89.

8 Schievink WI: Spontaneous dissection of the carotid and vertebral arteries. N Eng J Med 2001;344:898-906.

-9 Shaw JL: Bilateral posterior fracture-dislocation of the shoulder and other trauma caused by convulsive seizures. J Bone Joint Surg 1971;53:1437-1440.

10 Copuroglu C, Ozcan M, Dülger H, Yalniz E: Late-diagnosed bilateral intertrochanteric femur fracture during an epileptic seizure. Ulus Travma Acul Cerrahi Derg 2012;18:92-94.

11 Napier RJ, Nolan PC: Diagnosis of vertebral fractures in post-ictal patients. Emerg Med J 2011;28:169-170.

12 Yüksel HY, Hapa 0, Can M, Kürklü M: Bilateral simultaneous femoral neck fractures secondary to a postinfarct generalized tonic-clonic seizure. A case report. Hip Int 2010;20:287-291.

13 Copuroglu C, Aykac B, Tuncer B, Ozcan M, Yalniz E: Simultaneous occurrence of acute posterior shoulder dislocation and posterior shoulder-fracture dislocation after epileptic seizure. Int J Shoulder Surg 2009;3:49-51.

14 Tellisi NK, Abusitta GR, Fernandes RJ: Bilateral posterior fracture dislocation of the shoulders following seizure. Saudi Med J 2004;25:1727-1729.

15 Roohi F, Fox A: Burst fracture of the first lumbar vertebra and conus-cauda syndrome complications a single convulsive seizure: a challenge of diagnosis in the Emergency Department. J Emerg Med 2006;31:381-385.

16 Kumar SK, Freeman BJ: Quadriplegia following grand mal seizures. Injury 1999;30:626-629.

17 Albuquerque FC, Hu YC, Dashti SR, Abla AA, Clark JC, Alkire B, Theodore N, McDougall CG: Craniocervical arterial dissections as sequelae of chiropractic manipulation: patterns of injury and management. J Neurosurg 2011;115:1197-1205.

18 Reuter U, Hämling M, Kavuk I, Einhäupl KM, Schielke E: Vertebral artery dissections after chiropractic neck manipulation in Germany over three years. J Neurol 2006;253:724-730.

19 Buck D, Baker GA, Jacoby A, Smith DF, Chadwick DW: Patients' experiences of injury as a result of epilepsy. Epilepsia 1997;38:439-444.

20 Blandin CF, Norris JW: Stroke and seizures/epilepsy; in Welch KMA, Caplan LR, Reis DJ, Sisjö BK, Weir B (eds): Primer on Cerebrovascular Diseases. New York, Academic Press, 1997, pp. 355-360.

-21 Ferro JM, Canhão P, Bousser MG, Stam J, Barinagarrementeria F; ISCVT Investigators: Early seizures in cerebral vein and dural sinus thrombosis: risk factors and role of antiepileptics. Stroke 2008;39:1152-1158.
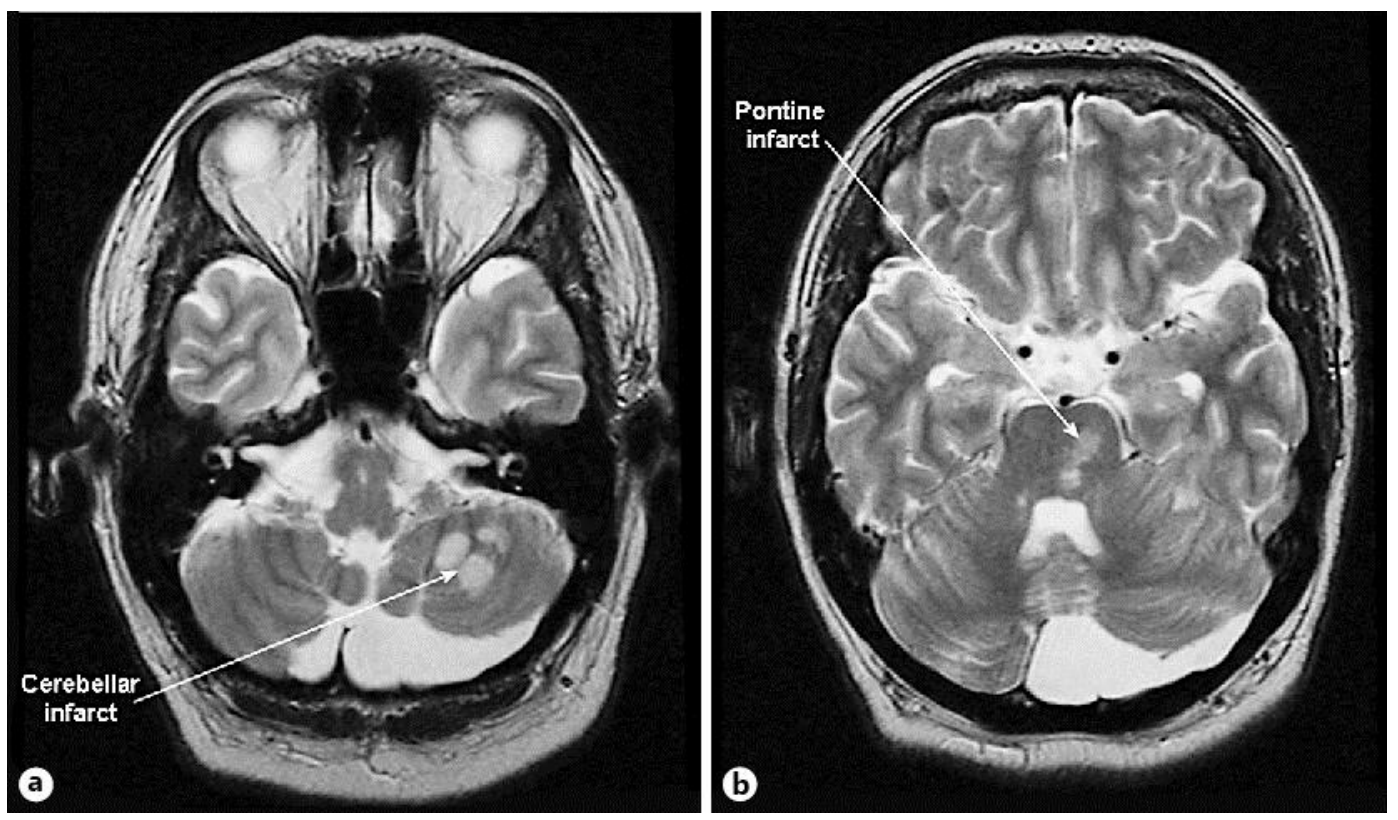

Fig. 1. T2-weighted axial turbo spin echo images showing the cerebellar (a) and pontine (b) infarcts. 
Amin et al.: Vertebral Artery Dissection Associated with Generalized Convulsive Seizures: A Case Report
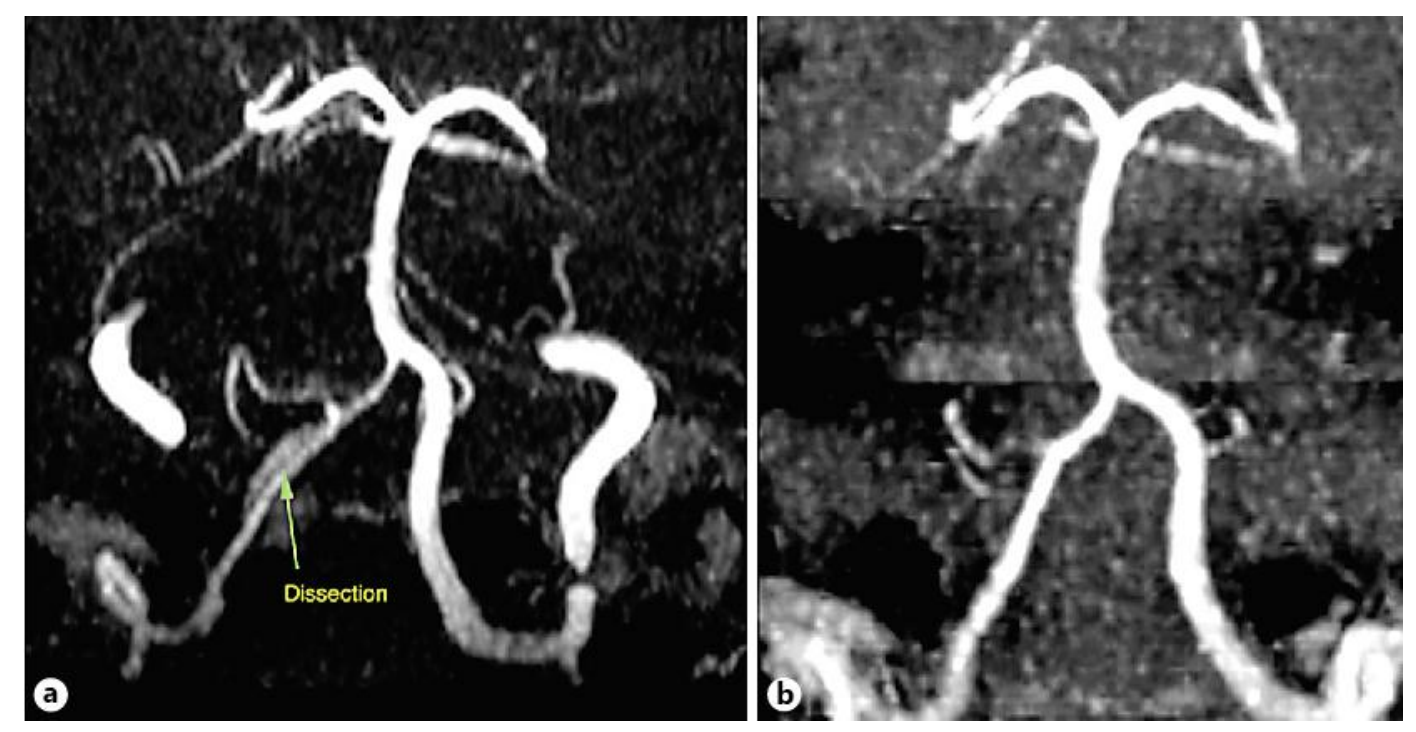

Fig. 2. a MR angiography demonstrating a right VAD 6 days after admission for a generalized convulsion. b Normal MR angiography 6 months later. 